\title{
4D GPS water vapor tomography New parameterized approaches
}

\section{Journal Article}

\section{Author(s):}

Perler, Donat; Geiger, Alain; Hurter, Fabian

Publication date:

2011-08

Permanent link:

https://doi.org/10.3929/ethz-b-000037202

\section{Rights / license:}

In Copyright - Non-Commercial Use Permitted

\section{Originally published in:}

Journal of Geodesy 85(8), https://doi.org/10.1007/s00190-011-0454-2 


\title{
4D GPS water vapor tomography: new parameterized approaches
}

\author{
Donat Perler · Alain Geiger · Fabian Hurter
}

Received: 15 June 2010 / Accepted: 18 February 2011 / Published online: 8 March 2011

(C) Springer-Verlag 2011

\begin{abstract}
Water vapor is a key variable in numerical weather prediction, as it plays an important role in atmospheric processes. Nonetheless, the distribution of water vapor in the atmosphere is observed with a coarse resolution in time and space compared to the resolution of numerical weather models. GPS water vapor tomography is one of the promising methods to improve the resolution of water vapor measurements. This paper presents new parameterized approaches for the determination of water vapor distribution in the troposphere by GPS. We present the methods and give first results validating the approaches. The parameterization of voxels (volumetric pixels) by trilinear and spline functions in ellipsoidal coordinates are introduced in this study. The evolution in time of the refractivity field is modeled by a Kalman filter with a temporal resolution of $30 \mathrm{~s}$, which corresponds to the available GPS-data rate. The algorithms are tested with simulated and with real data from more than 40 permanent GPS receiver stations in Switzerland and adjoining regions covering alpine areas. The investigations show the potential of the new parameterized approaches to yield superior results compared to the non parametric classical one. The accuracy of the tomographic result is quantified by the inter-quartile range (IQR), which is decreased by $10-20 \%$ with the new approaches. Further, parameterized voxel solutions have a substantially smaller maximal error than the non parameterized ones. Simulations show a limited ability to
\end{abstract}

D. Perler $(\bowtie) \cdot$ A. Geiger $\cdot$ F. Hurter

Geodesy and Geodynamics Laboratory, Institute of Geodesy and

Photogrammetry, ETH Zurich, Zurich, Switzerland

e-mail: donat.perler@geod.baug.ethz.ch

A. Geiger

e-mail: alain.geiger@geod.baug.ethz.ch

F. Hurter

e-mail: fabian.hurter@geod.baug.ethz.ch resolve vertical structures above the top station of the network with GPS tomography.

Keywords GPS · GPS meteorology - Water vapor · Tomography

\section{Introduction}

Urban growth and the advancing settlement of rural areas increase the vulnerability to natural hazards (Clark 2002). It is therefore of utmost interest to better understand the impact of global climate change on natural hazards and to improve hazard mitigation (Pilon 2005). In recent years, studies have been carried out to investigate the extent and the occurrence of natural hazards such as floods and landslides with highly resolved climate simulations (see, for example Dankers et al. 2009). At the same time, high-resolution numerical weather prediction models have been employed for natural hazard mitigation (Falconer et al. 2009; Ranzi et al. 2009). To validate and to initialize such models, appropriate meteorological observations are needed (Jacob et al. 2007; Buzzi 2008; Clark 2009). GPS water vapor tomography is a promising method providing information on the four-dimensional distribution of the water vapor content in the troposphere.

GPS tomography is a technique providing water vapor contents with high spatial and temporal resolution. Its ability has been demonstrated in numerous studies using GPS slant delays (Flores et al. 2000, 2001; Hirahara 2000; Nilsson 2005) and double differences (Troller et al. 2002, 2006; Lutz et al. 2010). Several studies indicate the limitations in vertical resolution (Troller 2004; Champollion et al. 2005; Bastin et al. 2007). Long-term studies have shown systematic differences between balloon soundings and tomographic solutions, and between solutions computed by a numerical weather 
model and tomographic solutions (Troller et al. 2007). These authors suggested that the differences are due to the geometry of the receiver station network. Investigations of highresolution tomography in mountainous regions have shown that better results can be achieved by refining the vertical discretization (Lutz 2009). However, higher resolution increases the computational costs and the influence of inter voxel (volumetric pixel) constraints on the results. The latter may introduce unintentional artifacts. The parameterization of voxels is an approach that reduces the effects of discretization and negligibly increases the computational costs. Moreover, the interpolation of in situ measurements to voxel central points becomes obsolete.

In this study, we introduce two new parameterizations of voxels in the Kalman filter-based GPS tomography software AWATOS 2 and their treatment in ellipsoidal coordinates. In addition, inter voxel constraints are presented. The new algorithms are investigated with simulated and real data over a mountainous region covering approximately $50,000 \mathrm{~km}^{2}$. The focus lies on the specific characteristics of different voxel parameterizations. The results of the simulations are discussed in terms of accuracy and the solutions computed with real data are compared with measurements of balloon soundings. The question about the vertical resolvability of GPS tomography is addressed and debated using simulated data.

\section{Preprocessing of the GPS data}

GPS satellites transmit at two or more frequencies in L-band. On the way from the satellite to the receiver located on the Earth's surface, the rays are considerably influenced by the atmosphere. There are atmospheric effects on the signals that are related to the propagation of the signal through the ionosphere and troposphere. The ionospheric effect can be eliminated to a large extent by combining the carrier frequencies due to the dispersive propagation of microwaves in the ionosphere. The second effect is induced by refractivity in the troposphere and cannot be eliminated by combining frequencies due to the non-dispersive behavior for frequencies below $30 \mathrm{GHz}$. Hence, an estimation process is needed and is taken into account by modern GPS processing software. Usually, one distinguishes between the slowly varying delays over time caused by dry air and the rapidly varying ones by water vapor. The relation between the meteorological description of the atmosphere (partial pressure of dry air $p_{d}$, partial pressure of water vapor $p_{w}$, and temperature $T$ ) and refractivity can be characterized by the formula published in Rüeger (2002)

$$
N_{\text {tot }}=\left(n_{\text {tot }}-1\right) 10^{6}=\underbrace{77.6890 \frac{p_{d}}{T}}_{\text {dry part }}+\underbrace{71.2952 \frac{p_{w}}{T}+375463 \frac{p_{w}}{T^{2}}}_{\text {wet part }},
$$

where $N_{\text {tot }}$ is the total refractivity in (ppm) with refractive index $n_{\text {tot }}$. The unit of partial pressure is $\mathrm{hPa}$ and that of temperature $T$ is $\mathrm{K}$. The propagation delay $\Delta^{P D}$ from the satellite to the receiver is defined by the integral of the refractivity $N$ along the ray path from satellite $r$ to receiver $q$ :

$\Delta_{q}^{P D, r}=10^{-6} \int_{r}^{q} N \mathrm{~d} s$

The Bernese GPS Software Version 5.0 (Dach et al. 2007) is used to estimate the tropospheric path delays. For each station, the time series of the zenith path delay (ZPD) are modeled by piecewise linear functions. Thereby, a mapping function relates a slant path delay to a ZPD. A fairly large number of mapping functions have been developed by different authors. For the real data experiment in this study, the setup of the operational processing at the Swiss Federal Office of Topography was used. In this setup, Niell's mapping functions are used (Niell 1996) neglecting the effect of the difference between the dry and hydrostatic part on the parameters of the mapping function (Troller 2004; Dach et al. 2007).

The double difference residuals used in this study have been provided by the Swiss Federal Office of Topography swisstopo using the Bernese GPS Software. A double difference is built by four slant path delays from satellites $r$ and $s$ to stations $p$ and $q$.

$$
\Delta_{p q}^{2, P D, r s}=\left(\Delta_{q}^{P D, r}-\Delta_{p}^{P D, r}\right)-\left(\Delta_{q}^{P D, s}-\Delta_{p}^{P D, s}\right) .
$$

The tomography software uses double differences of wet path delays as input data to compute the refractivity field. They can be computed from the output of Bernese GPS Software and meteorological parameters at the GPS receiver stations in four steps:

1. Compute the isotropic part of the total slant path delays $\overline{\Delta_{\text {tot }, p}^{P D, r}}$ from the ZPD $\overline{\Delta_{\text {apr }, p}^{P D}}$ corresponding to the a priori model, and ZPD $\overline{\Delta_{\text {corr }, p}^{P D}}$ corresponding to the correction model according to

$\overline{\Delta_{\text {tot }, p}^{P D}}=\overline{\Delta_{\text {apr }, p}^{P D}} \cdot \mathrm{m}_{\text {apr }}\left(\varphi_{p}^{r}\right)+\overline{\Delta_{\text {corr }, p}^{P D}} \cdot \mathrm{m}_{\text {corr }}\left(\varphi_{p}^{r}\right)$,

where the ZPDs $\overline{\Delta_{\text {apr }, p}^{P D}}$ and $\overline{\Delta_{\text {corr, } p}^{P D}}$ are estimated by the Bernese GPS Software using mapping functions $\mathrm{m}_{\text {apr }}\left(\varphi_{p}^{r}\right)\left(\right.$ dry Niell) and $\mathrm{m}_{\text {corr }}\left(\varphi_{p}^{r}\right)($ wet Niell) with elevation angle $\varphi_{p}^{r}$ at station $p$ to satellite $r$. The overline denotes the isotropic delays.

2. Eliminate the dry part of the slant path delay by applying a modified version of Saastamoinen's formula $\mathrm{s}\left(p_{d}, T, \varphi_{p}^{r}, h_{p}\right)$ (Saastamoinen 1972; Troller 2004). For this step, meteorological data at the receiver stations 
and their altitudes are needed. For GPS stations which are not equipped with meteorological sensors, the missing data are interpolated by the collocation approach (Troller 2004). The isotropic part of the wet slant path delay is computed by

$\overline{\Delta_{\mathrm{wet}, p}^{P D, r}}=\overline{\Delta_{\mathrm{tot}, p}^{P D, r}}-\mathrm{s}\left(p_{d}, T, \varphi_{p}^{r}, h_{p}\right)$.

3. Build the isotropic double differences of the wet path delays from the isotropic wet slant path delays using Eq. 3.

4. Add the double difference residuals $\Delta_{p q}^{2, R E S, r s}$ from the processing with Bernese GPS Software. As in Flores et al. (2000) and Gradinarsky and Jarlemark (2004), Troller et al. (2006), we assume that the residuals are dominated by the effect of the non-isotropic distribution of the wet refractivity.

$$
\Delta_{\mathrm{wet}, p q}^{2, P D, r s}=\overline{\Delta_{\mathrm{wet}, p q}^{2, P D, r s}}+\Delta_{p q}^{2, R E S, r s}
$$

\section{Modeling the wet refractivity field with parameterized GPS tomography}

The wet refractivity field is discretized along the axes longitude $\lambda$, latitude $\phi$ and height $h$ with reference to the WGS84 ellipsoid and the current state of the field is denoted by the vector $\mathbf{x}$. The time evolution of the field is modeled as random walk

$\dot{\mathbf{x}}=\mathbf{w}$,

where $\mathbf{w}$ is the process noise. The noise, describing the prediction error within a prediction step, is multivariate normally distributed with covariance matrix $\mathbf{Q}$. To be capable of sequentially assimilating observations, we use the Kalman filter approach (see Gelb 1974) with prediction step from time $t_{m-1}$ to $t_{m}$

$\hat{\mathbf{x}}_{m \mid m-1}=\mathbf{F}_{m} \hat{\mathbf{x}}_{m-1 \mid m-1}$

$\mathbf{P}_{m \mid m-1}=\mathbf{F}_{m} \mathbf{P}_{m-1 \mid m-1} \mathbf{F}_{m}^{T}+\mathbf{Q}_{m}$

and update step

$\hat{\mathbf{x}}_{m \mid m}=\hat{\mathbf{x}}_{m \mid m-1}+\mathbf{K}_{m}\left(\mathbf{z}_{m}-\mathbf{H}_{m} \hat{\mathbf{x}}_{m \mid m-1}\right)$

$\mathbf{P}_{m \mid m}=\left(\mathbf{I}-\mathbf{K}_{m} \mathbf{H}_{m}\right) \mathbf{P}_{m \mid m-1}$

with the Kalman gain

$\mathbf{K}_{m}=\mathbf{P}_{m \mid m-1} \mathbf{H}_{m}^{T}\left(\mathbf{H}_{m} \mathbf{P}_{m \mid m-1} \mathbf{H}_{m}^{T}+\mathbf{R}_{m}\right)^{-1}$

where $\hat{\mathbf{x}}_{m-1 \mid m-1}$ represents the estimated refractivity field at time $t_{m-1}$, and $\mathbf{P}_{m-1 \mid m-1}$ its covariance matrix. The matrix $\mathbf{F}_{m}$ propagates the field one time step forward and $\mathbf{Q}_{m}$ reflects the uncertainty of the propagation. In the update step, the predicted state $\hat{\mathbf{x}}_{m \mid m-1}$ is mapped by the observation matrix $\mathbf{H}_{m}$ into the space of the observations. $\mathbf{z}_{m}$ denotes the observation vector and $\mathbf{R}_{m}$ the covariance matrix of the observations.

Modelling a Gaussian random walk (see Eq. 4), the propagation matrix $\mathbf{F}_{m}$ is equal to the identity matrix. The elements of the process noise matrix $\mathbf{Q}_{m}$ are empirically determined using measurements from balloon soundings. Data of 68 European balloon sounding stations over a period of 2 years were analyzed with a conventional autocovariance estimation process (Perler 2011). In the update step, a noise of $5 \mathrm{~mm}$ is assumed on the GPS ZPDs (see, e.g. Nilsson and Gradinarsky 2006). This uncertainty is taken into account by the covariance matrix of the observations $\mathbf{R}_{m}$.

\subsection{Parameterized voxels}

There are several ways to discretize a three dimensional field. Our software provides three different approaches:

\section{Constant parameterization}

This is the simplest of the provided approaches. The refractivity is constant within a voxel. Thus, one parameter per voxel must be estimated in the Kalman filter. In the following sections, we will refer to this approach as non-parametric or as constant.

\section{Trilinear parameterization}

In the trilinear parameterization, the refractivity at the point $(\lambda, \phi, h)$ is determined by a weighted sum of the 8 refractivity values at the corners of the voxel, in which the point is located. The interpolation function is defined by

$\mathrm{N}(\lambda, \phi, h)=\omega^{T} \mathbf{N}_{\text {voxel }, i, j, k}$

with

$\boldsymbol{\omega}=\left[\begin{array}{ccc}\frac{\lambda_{i+1}-\lambda}{\lambda_{i+1}-\lambda_{i}} & \frac{\phi_{j+1}-\phi}{\phi_{j+1}-\phi_{j}} & \frac{h_{k+1}-h}{h_{k+1}-h_{k}} \\ \frac{\lambda-\lambda_{i}}{\lambda_{i+1}-\lambda_{i}} & \frac{\phi_{j+1}-\phi}{\phi_{j+1}-\phi_{j}} & \frac{h_{k+1}-h}{h_{k+1}-h_{k}} \\ & \vdots & \\ \frac{\lambda-\lambda_{i}}{\lambda_{i+1}-\lambda_{i}} & \frac{\phi-\phi_{j}}{\phi_{j+1}-\phi_{j}} & \frac{h-h_{k}}{h_{k+1}-h_{k}}\end{array}\right]$

$\mathbf{N}_{\text {voxel }, i, j, k}=\left[\begin{array}{c}N_{i, j, k} \\ N_{i+1, j, k} \\ \vdots \\ N_{i+1, j+1, k+1}\end{array}\right]$

In contrast to the first parameterization type, the parameters to be estimated are located at the corners of the voxels. Consequently, there are slightly more parameters to estimate $\left(n_{\lambda}+1\right)\left(n_{\phi}+1\right)\left(n_{h}+1\right)$ instead of $n_{\lambda} n_{\phi} n_{h}$ where $n_{\lambda}$ is the number of voxels in longitudinal, $n_{\phi}$ in latitudinal, and $n_{h}$ in the vertical direction, respectively. In figures, we will often use the term linear for this approach. 


\section{Bilinear/spline parameterization}

This approach uses direction dependent parameterization techniques. In the horizontal directions $\lambda$ and $\phi$, the bilinear form is used and, in the vertical one, natural splines (Schwarz 1997). The refractivity is then interpolated from values on the four adjoining vertical profiles. The refractivity at any point can be expressed by the refractivities and their second derivatives in the vertical direction at the corners of the corresponding voxel.

$\mathrm{N}(\lambda, \phi, h)=\omega^{T}\left[\begin{array}{l}\mathrm{N}_{k}(\lambda, \phi) \\ \mathrm{N}_{k+1}(\lambda, \phi) \\ \mathrm{N}_{k}^{\prime \prime}(\lambda, \phi) \\ \mathrm{N}_{k+1}^{\prime \prime}(\lambda, \phi)\end{array}\right]$

with

$\boldsymbol{\omega}=\left[\begin{array}{c}1-\frac{h-h_{k}}{d h_{k}} \\ \frac{h-h_{k}}{d h_{k}} \\ \frac{\left(h-h_{k}\right)^{2}}{2}-\frac{d h_{k}\left(h-h_{k}\right)}{3}-\frac{\left(h-h_{k}\right)^{3}}{6 d h_{k}} \\ \frac{\left(h-h_{k}\right)^{3}}{6 d h_{k}}-\frac{d h_{k}\left(h-h_{k}\right)}{6}\end{array}\right]$,

where $d h_{k}$ is the thickness of the $k$ th layer. The $\mathrm{N}_{k}(\lambda, \phi)$ and $\mathrm{N}_{k}^{\prime \prime}(\lambda, \phi)$ are the bilinearly interpolated refractivity and second derivative of the refractivity at height $h_{k}$, respectively. Note that $\omega$ is independent of $\lambda$ and $\phi$.

The vector $\mathbf{N}_{i, j}^{\prime \prime}$ containing the second derivatives along a vertical profile at $\left(\lambda_{i}, \phi_{j}\right)$ can be expressed by refractivity values along the same profile using the spline condition

$\mathbf{C N}_{i, j}^{\prime \prime}=-\mathbf{D N} \mathbf{N}_{i, j}$

with

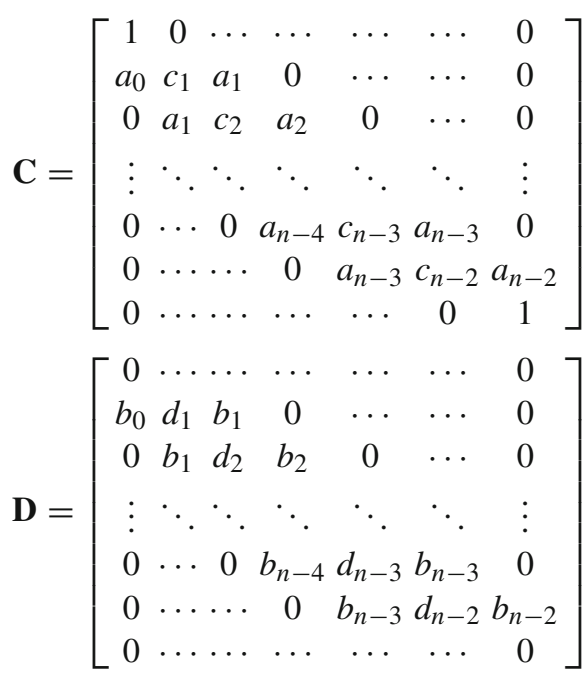

where $a_{k}=d h_{k}, b_{k}=\frac{6}{d h_{k}}, c_{k}=2\left(d h_{k-1}+d h_{k}\right)$ and $d_{k}=$ $-\frac{6}{d h_{k-1}}-\frac{6}{d h_{k}}$. Consequently, the second derivatives $N_{k}^{\prime \prime}$ and $N_{k+1}^{\prime \prime}$ in Eq. 11 can be expressed by the refractivity vector $\mathbf{N}_{i, j}$ and, thus, the field of the second derivatives does not have to be stored. The same number of parameters have to be estimated as in the case of the trilinear approach. Since
$\mathbf{C}$ as well as $\mathbf{D}$ are the same for any $\left(\lambda_{i}, \phi_{j}\right)$ and the grid geometry does not change over time, the matrix $-\mathbf{C}^{-1} \mathbf{D}$ has to be computed only once. In figures, we often refer to this method as spline.

\subsection{Implementation of the observations in a parameterized field}

With the above equations, point observations can be directly introduced into the update step of the Kalman filter. How can integral observations like double-differences be taken into account under the different parameterizations? For the nonparametric approach, a slant wet delay as defined by Eq. 2 is described by the sum of the distances of a ray $\Delta s_{i}$ crossing the voxels along its path multiplied by the refractivity $N_{\text {wet, } i}$ of the voxel $i$ :

$\Delta_{\text {wet }}^{P D}=10^{-6} \sum_{i} N_{\text {wet }, i} \Delta s_{i}$.

In the parameterized cases, the discretization becomes more difficult. The path is again subdivided into sections corresponding to voxels.

$\Delta_{\text {wet }}^{P D}=10^{-6} \sum_{i} \int_{s_{i}}^{s_{i+1}} \mathrm{~N}_{\text {wet }, i}(s) \mathrm{d} s$.

The integral in Eq. 14 does generally not exist in closed form because the path of the ray in ellipsoidal coordinates cannot be expressed analytically in most cases. Therefore, the integrals are solved by Newton-Cotes quadrature (see Appendix A). This allows the integral in Eq. 14 to be expressed as a weighted sum of the refractivities at the grid nodes. Independent of the parameterization type, four slant delay equations (Eq. 14) are finally combined into a double difference observation according to Eq. 3. The resulting double difference observation equation is a weighted sum of refractivities. These weights go into the observation matrix $\mathbf{H}_{m}$ and, hence, into the update step of the Kalman filter.

Besides the double difference observations, pseudo observations describing inter voxel relations might be introduced to smooth and stabilize the update process (Ruffini et al. 1998). We apply inter-voxel constraints differently than Ruffini et al. (1998). Instead of smoothing over all neighbors of a voxel, we constrain the refractivity variation only in the horizontal directions by

$\frac{\partial N}{\partial \lambda}=0$
$\frac{\partial N}{\partial \phi}=0$.

These constraints are introduced as weighted pseudoobservations in the calculation. No restrictions are made in the vertical direction. GPS tomography has a rather weak 
resolution in the vertical direction due to no tangentially propagating rays with respect to the Earth's surface. Constraining in the vertical would decrease the influence of the double difference observations on the already weakly determined vertical resolution. Further investigations are in progress to assess inter voxel constraints in more detail.

\section{Results from experiments with synthetic and real data}

We have carried out several tests to assess the new tomographic algorithms using GPS double difference observations. In a first part, we present results based on simulated measurements and in a second part real observations are analyzed. For both parts, the same GPS station configuration is used.

\subsection{Network and voxel model}

The stations belong to the AGNES (Automated GPS Network for Switzerland) operated by the Swiss Federal Office of Topography swisstopo, or to the EPN (EUREF Permanent Network) operated by EUREF. The AGNES consists of 31 stations, and 15 additional stations belong to the EPN (see Fig. 1). The AGNES stations are situated in the core area of investigation and have a mean shortest inter station distance of about $30 \mathrm{~km}$. The EPN is much coarser than AGNES and its stations are located towards the margin of the investigation area. The stations of both networks are at many different altitudes ranging from $310 \mathrm{~m}$ up to $3,584 \mathrm{~m}$ above the reference ellipsoid (see Fig. 2).

The area of investigation ranges from $6.5^{\circ}$ to $9.5^{\circ}$ in longitude, $46^{\circ}$ to $47.5^{\circ}$ in latitude, and $0-15,000 \mathrm{~m}$ in WGS 84 ellipsoidal height. In addition, the core area is surrounded by a fringe of $10^{\circ}$ to ensure that no ray path crosses the lateral

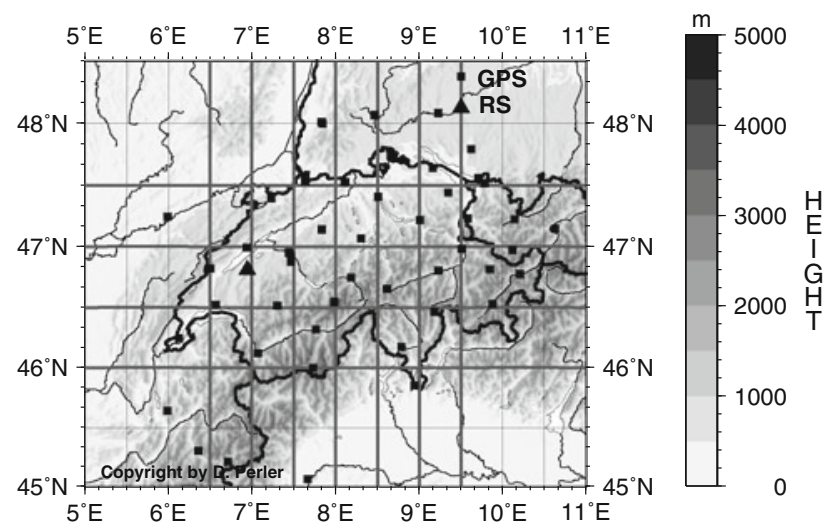

Fig. 1 Map covering the core area of investigation with GPS stations (black squares) and radio sounding station RS at Payerne (black triangle). The gray lines indicate the floor plan of the grid used in the tomographic processing

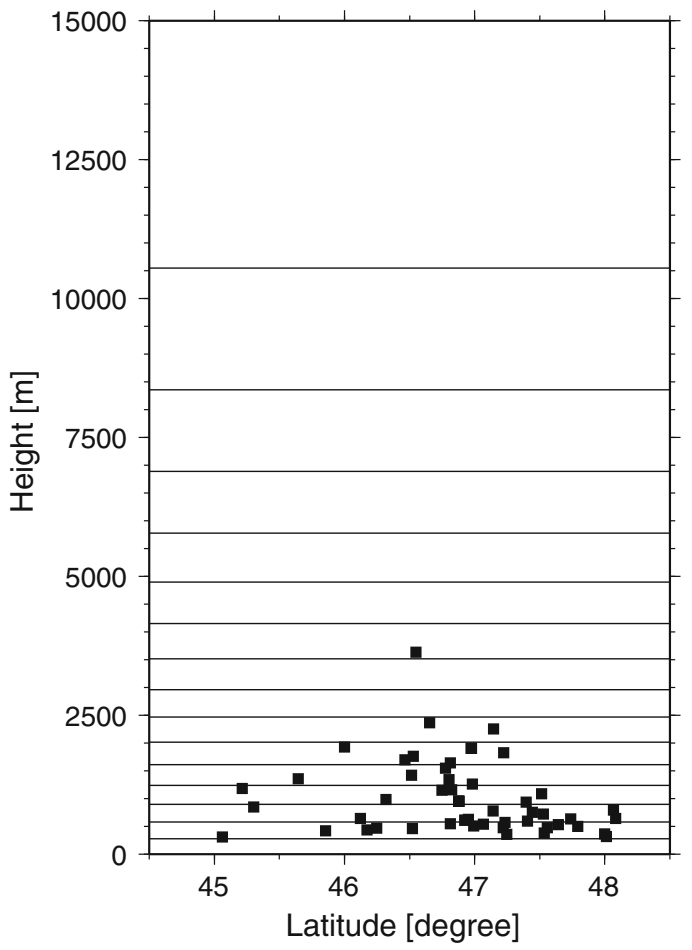

Fig. 2 Height distribution of the GPS stations (black squares) and the levels of the ellipsoidal voxel model used in the tomographic processing

boundaries of the model area. The horizontal discretization is $0.5^{\circ}$, which corresponds to a horizontal resolution of about $37 \mathrm{~km}$. The thickness of the layers increases with height (see Fig. 2).

\subsection{Experiments with synthetic data}

We investigate the question of how well the three parameterizations (constant voxel, trilinear, bilinear/spline) can approximate two synthetic atmospheric patterns. The patterns vary only in the vertical direction. This choice is motivated by the fact that the resolution of vertical structures is much more challenging in GPS tomography than horizontal ones (see Bastin et al. 2007). Horizontal variations are not considered even though they are certainly present in the atmosphere within the area of interest. The first pattern is an average refractivity profile computed from data of weather balloons launched at Payerne. The motivation for this experiment is to see how quickly the algorithms converge and if they show systematic errors. The second experiment is a spike layer test. The proper reconstruction of sharp vertical refractivity changes is of importance, since they can occur during inversions. Inversions are an atmospheric pattern occasionally observed in the Swiss plains during winter (Wanner 1979). By varying the height and the thickness of the spike layers, the vertical resolution capabilities of the tomographic algorithms are investigated. 
For the average profile, the following exponential function with refractivity $N$ and height $h$ has been fitted to balloon soundings:

$\mathrm{N}(h)=N_{0} \exp \left(-\frac{h}{h_{s}}\right)$

with the empirically determined parameters $N_{0}=77.5 \mathrm{ppm}$ and $h_{s}=2,178 \mathrm{~m}$. Given a wet refractivity field generated by this function, we simulated double difference observations with a sampling rate of $30 \mathrm{~s}$ using a ray tracing algorithm. The ray tracer integrates the refractivity along the geometrical path and uses Newton-Cotes quadrature (see Jehle et al. 2008 for more details). On each ray, normally distributed noise of $5 \mathrm{~mm}$ in zenith direction is added. The double difference observations are the only observations used in this simulation and no inter voxel constraints are involved in these experiments. The duration of the simulated period corresponds to the full cycle of the GPS satellite constellation (approximately one day) from November 26, 2006. The Kalman filter was initialized with a uniform zero refractivity.

We evaluated the tomographic solutions along the profile ranging from $600 \mathrm{~m}$ (approximately Earth's surface) to $15,000 \mathrm{~m}$ above the reference ellipsoid and located at $47.0^{\circ} \mathrm{N}$ and $8.5^{\circ} \mathrm{E}$. The profile is situated in the core area of investigation, but not in the high mountains. This allows us to analyze the troposphere in the lower altitudes, too. Note that the refractivity in the voxel column ranging from 8.0 to $8.5^{\circ} \mathrm{E}$ and 46.5 to $47^{\circ} \mathrm{N}$ is evaluated for the constant parameterized approach. The refractivities in the other three voxel columns adjoining $8.5^{\circ} \mathrm{E}$ and $47.0^{\circ} \mathrm{N}$ do not significantly differ. Figure $3 \mathrm{a}-\mathrm{c}$ shows solutions along this profile for different parameterizations. Solutions are plotted every 5 min with different grey shades beginning with light grey. The solutions of all three parameterizations approximate well the model profile already after about $15 \mathrm{~min}$. However, the limitation of the non-parametric approximation can be clearly seen in Fig. 3d. The errors become as high as $3.8 \mathrm{ppm}$ in the lower troposphere and decrease with height. The maximal errors are obviously smaller for the two parameterized solutions, 1.1 and $0.7 \mathrm{ppm}$ for the trilinear and the bilinear/spline case, respectively (see Fig. 3e, f). Considering the entire profile, the standard errors are again significantly smaller for the parameterized solutions than for the non-parameterized one (see Table 1).

For the spike layer tests, the same parameter settings are used as for the first test (sample rate of $30 \mathrm{~s}$, noise level of $5 \mathrm{~mm}$, simulation duration of $24 \mathrm{~h}$, and initialization of the Kalman filter with a uniform zero refractivity). The given model atmosphere is everywhere 0 ppm except within one layer, where the refractivity is $3.5 \mathrm{ppm}$. This value corresponds to the accuracy achieved by in situ measurements (see Flores et al. 2000). At a spike layer thickness of $200 \mathrm{~m}$, such vertical perturbations cause a change in the measured tropospheric delays of the same order as the noise level of a single path delay observations. We investigated spike layer thicknesses of 200, 400, and $800 \mathrm{~m}$ located at different heights. The lower bound of the layers are at $500 \mathrm{~m}$ up to 4,000 $\mathrm{m}$ in $500 \mathrm{~m}$ steps and, additionally, one on 5,000 $\mathrm{m}$ and one on 6,000 $\mathrm{m}$ above the reference ellipsoid. Evaluations are carried out along the same profile as in the previous test. Examining other profiles yielded similar results.

The thickness of the spike layer in Fig. 4 approximately corresponds to the height resolution of the grid at this altitude. The discontinuities of the spike layer function cannot be exactly resolved by the parameterized voxel modeling. In contrast, the non-parameterized representation is capable to reproduce them exactly as long as the discontinuities are at the borders of a voxel. On the one hand, the nonparameterized approach smoothes out the spike layer much more than the parameterized ones. On the other hand, the parameterized approaches show much clearer negative deflections below and above the layer than in the non-parameterized case. The maximum peak is aligned with voxels in the solutions of the non-parametric approach and is shifted to voxel boundaries in the trilinear parameterization. Contrary, the peak matches very well the middle height of the layer in the bilinear/spline case. This is one of the major advantages of the bilinear/spline parameterization over the two others.

A more quantitative view of the accuracies is achieved by evaluating the refractivity along the vertical test profile ranging from $600 \mathrm{~m}$ to 15,000 altitude at $47.0^{\circ} \mathrm{N}$ and $8.5^{\circ} \mathrm{E}$. To assess the accuracy, the inter quartile range (IQR) was computed from the differences between the model atmosphere and the tomographic solution at points along the test profile. Each solution is evaluated at 2,960 points. A smaller IQR reflects better accuracy. Hypothesis testing is used to decide if the IQRs among the parameterizations are significantly different from each other. As the sample size is rather small (30 different spike layer profiles for each parameterization) and normal distribution cannot be assured, we used the Wilcoxon signed rank test (Rice 1995) with a significance level of 0.05 . The IQR of the bilinear/spline parameterized solutions is significantly smaller than the one from the trilinear parameterized and the non-parameterized solutions. The accuracy of the trilinear parameterized solutions is better than the one of the non-parameterized solutions; however, not as clear as in the previous comparisons.

How well a spike layer is resolved depends mainly on its height and its contribution to slant delays. Spike layers with a thickness of $800 \mathrm{~m}$ are well reproduced below 4,500 m (see Fig. 5a). Thinner spike layers are not. They are only well represented below 3,500 m, as shown by Fig. 5c. Our investigations show that an insufficient grid spacing is not a major cause for the poor retrieval of high altitude spike layers. Solutions computed with an equidistant grid of $200 \mathrm{~m}$ 


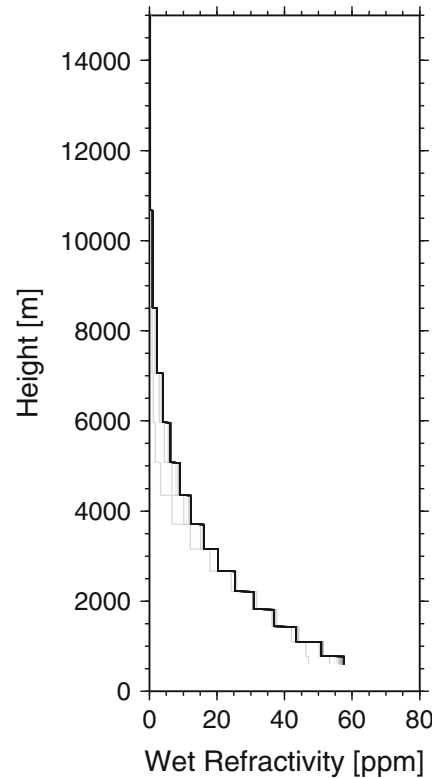

(a)

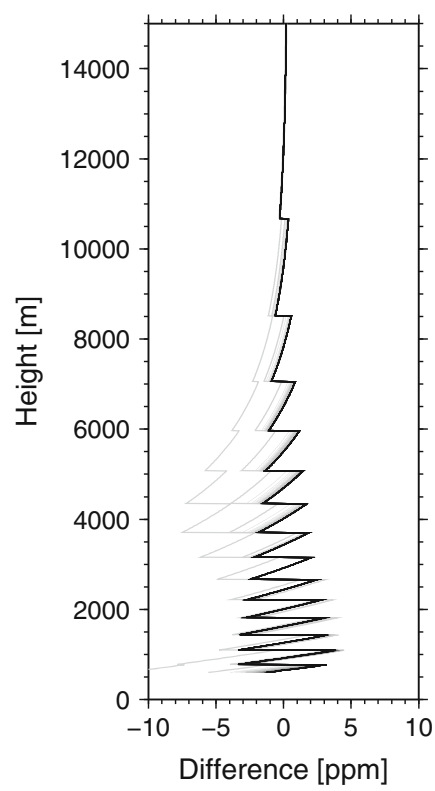

(d)

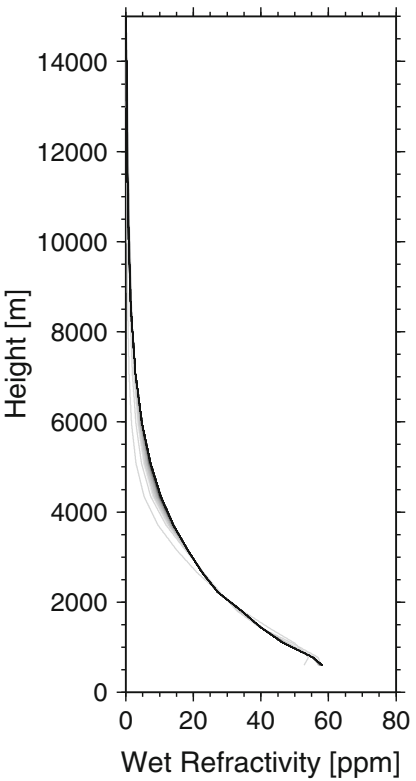

(b)

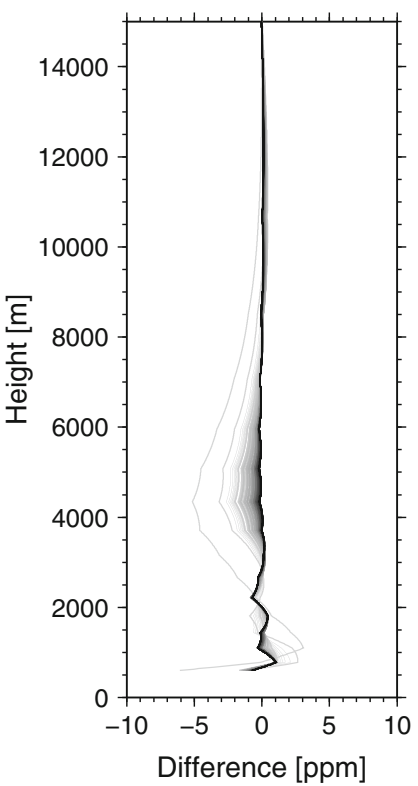

(e)

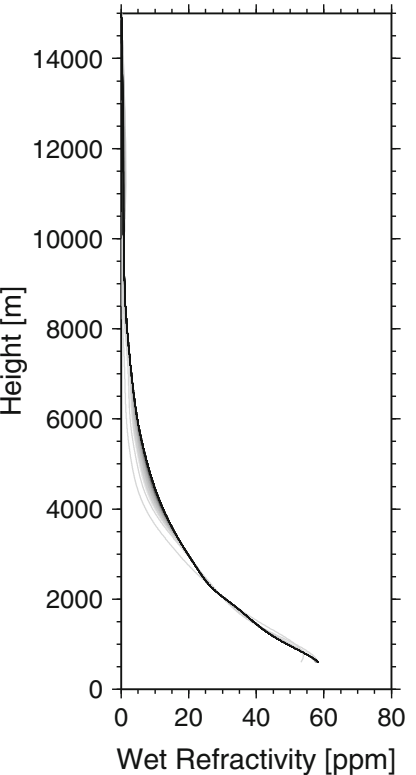

(c)

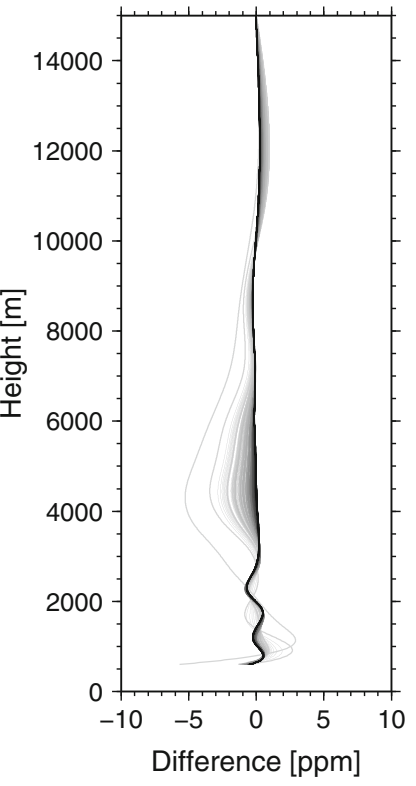

(f)
Fig. 3 Tomographic results using simulated double difference observations with different parameterizations along the vertical profile at $47.0^{\circ} \mathrm{N}$ and $8.5^{\circ} \mathrm{E}$ in refractivity units. a-c Refractivity profiles. $\mathbf{d}-\mathbf{f}$ Differences between retrieved profiles and the synthetic model profiles (computed minus model). Observations are generated from an atmo-

vertical resolution show the same characteristics as the solutions calculated with the grid displayed in Fig. 2. The IQRs shown in Fig. 5b do not remarkably differ from the ones in Fig. 5a although the finer grids have a much smaller grid resolution than the thickness of the spike layer. Even for a thinner spike layer of $200 \mathrm{~m}$, no obvious difference in accuracy is sphere with refractivities decreasing exponentially with height. Every 5 min, a profile is plotted starting from light grey shade at the beginning to black at the end of one simulation day. a, $\mathbf{d}$ The result with constant parameterization, $\mathbf{b}, \mathbf{e}$ with trilinear, and $\mathbf{c}, \mathbf{f}$ the hybrid of bilinear and spline parameterization observed between the two different grid configurations (see Fig. 5c, d).

The results plotted in Fig. 5a show that the layers below the top station at 3,584 m altitude (see Fig. 2) are well resolved. This is due to the good height distribution of the stations in the network. Furthermore, Fig. 5a shows two well-resolved 
Table 1 Statistical comparison of the three parameterizations based on simulated measurements

\begin{tabular}{llll}
\hline Parameterization & Mean & Std.error & Max. error \\
\hline Const & 0.010 & 0.871 & 3.832 \\
Trilinear & 0.008 & 0.176 & 1.060 \\
Bilinear/spline & 0.004 & 0.209 & 0.716 \\
\hline
\end{tabular}

Double difference observations were generated from an atmosphere where the refractivity decreases exponentially with height. The simulated data served as input for the tomographic software. The statistics is based on 1,441 points distributed uniformly along the vertical profile in the center of the area of investigation after one simulation day. All values are given in (ppm) refractivity units

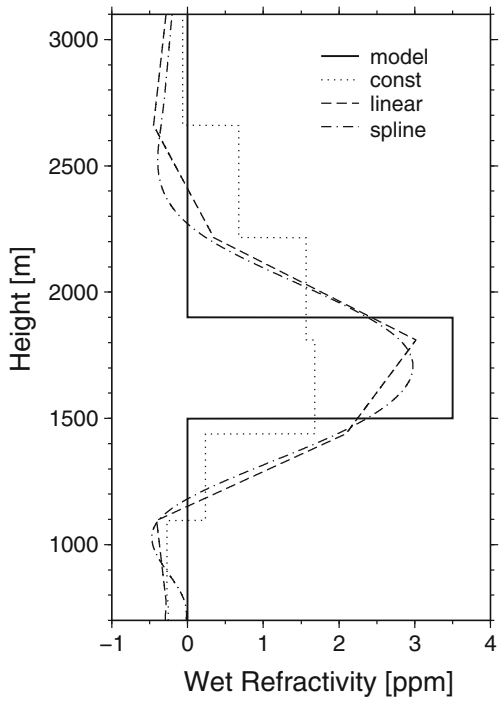

Fig. 4 Refractivity values along a profile. The solid line represents the given model atmosphere from which GPS double difference observations are generated. The spike has the lower bound at 1,500 m altitude and a thickness of $400 \mathrm{~m}$. The refractivity within the spike layer is 3.5 and $0 \mathrm{ppm}$ elsewhere. Using the simulated observations, the dotted line is the solution of the non-parameterized approach, the dashed one the solution of the trilinear approach and the dash-dotted the solution of the bilinear/spline approach. Note that neither vertical nor horizontal constraints are used

spike layers located above the top station between 3,500 and $4,500 \mathrm{~m}$ altitude. This indicates that also above the top station information about the vertical refractivity distribution can still be gained. First investigations suggest that this can be explained with the curvature of the ellipsoidal grid layer. At altitudes above $4,500 \mathrm{~m}$, the resolvability of perturbations in the vertical rapidly decreases.

The influence of the Earth's curvature in tomography depends on the spatial extent of the network. The smaller the area covered by the network the less the shell shaped layer differs from its local plane-shaped approximation above this area. As plane-shaped layers above the top station cannot be resolved by a typical measurement setup of GPS tomography, the resolvability of shell-shaped layers are more limited using networks with a small spatial extent than

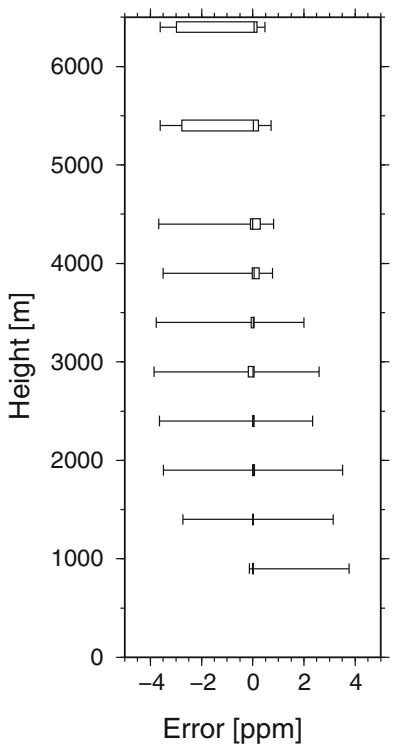

(a)

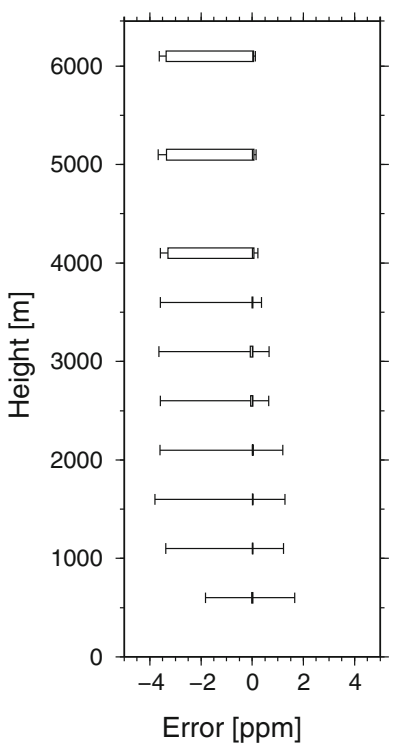

(c)

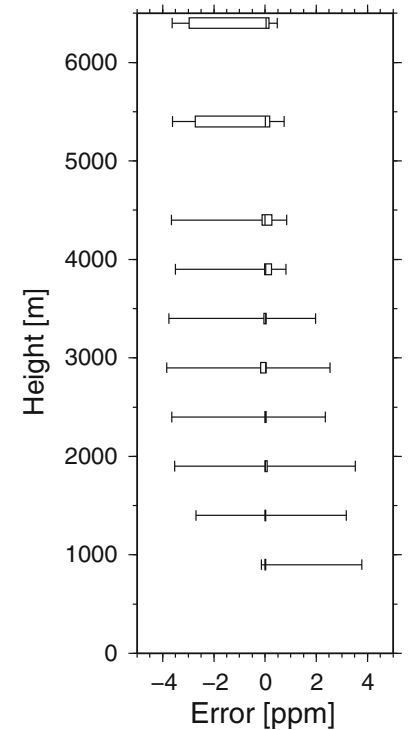

(b)

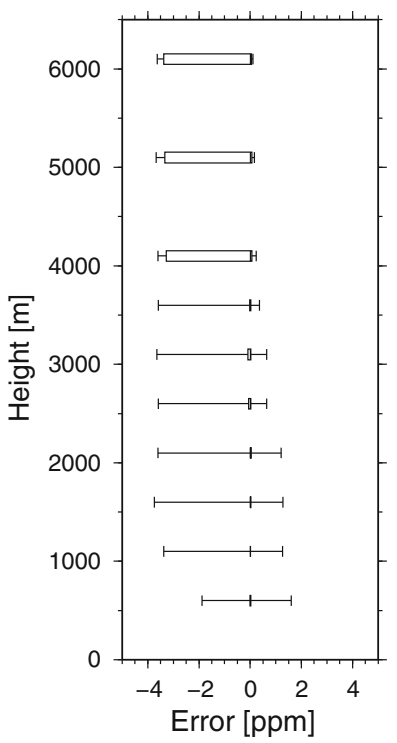

(d)
Fig. 5 Box plots of the difference between computed and modeled refractivities with different spike layer heights using the bilinear/spline parametrization. In the first row, a spike layer thickness of $800 \mathrm{~m}$ is given and in the second a thickness of $200 \mathrm{~m}$. The columns show two different grids. The first has exponential decreasing height resolution ranging from $280 \mathrm{~m}$ up to $4,320 \mathrm{~m}$ and a second has a constant height resolution of $200 \mathrm{~m}$

networks with a larger extent. As a consequence, the condition number of the observation matrix $\mathbf{H}$ is increased and, accordingly, measurement errors have a stronger influence on the accuracy of the estimated refractivities. Observations providing vertically resolved data make the largest contribution in improving the vertical resolution. Such observations could be included from balloon soundings, radio occulations or LIDARs. 


\subsection{Experiments using real network data}

Data from the GPS network shown in Fig. 1 were processed with the Bernese GPS Software Version 5.0 on the basis of double differences (see Dach et al. 2007). The wet part of the double differences are reconstructed using the esti- mated ZPDs, the residuals of the double difference processing, ground-based meteorological data, and the positions of the receivers and satellites, as described in Sect. 2. Besides the double difference observations, inter voxel pseudo observations as described in Sect. 3 by the Eqs. 15 and 16 are used in the tomographic processing with a weight of $60^{-2}$. The
Fig. 6 Comparison of two radio soundings (grey dots) with the tomographic solutions (black lines) of the different parameterization approaches. In the first row, data is compared at 2006-02-26 $0 \mathrm{~h}$ UTC and, in the second one, at 2006-02-26 $12 \mathrm{~h}$ UTC

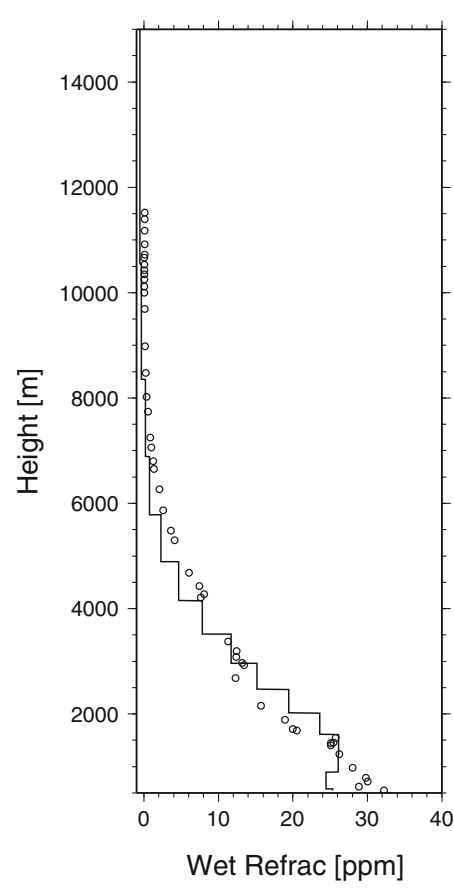

(a) const, at $00 \mathrm{~h}$ UTC

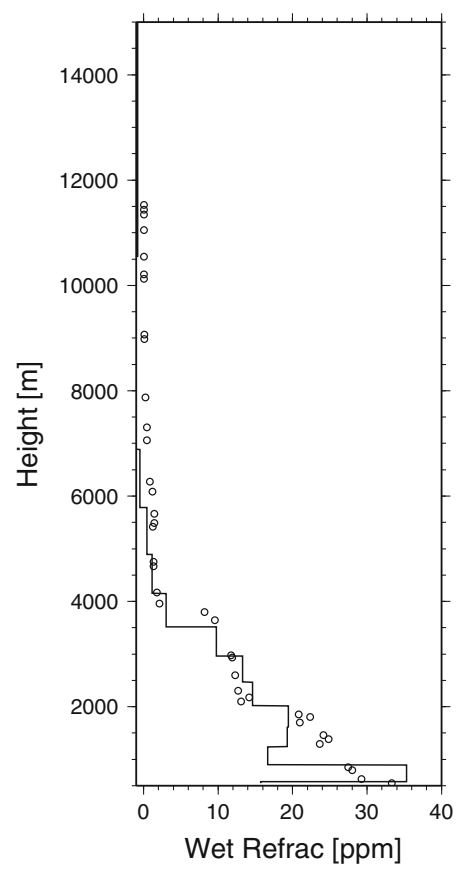

(d) const, at $12 \mathrm{~h}$ UTC

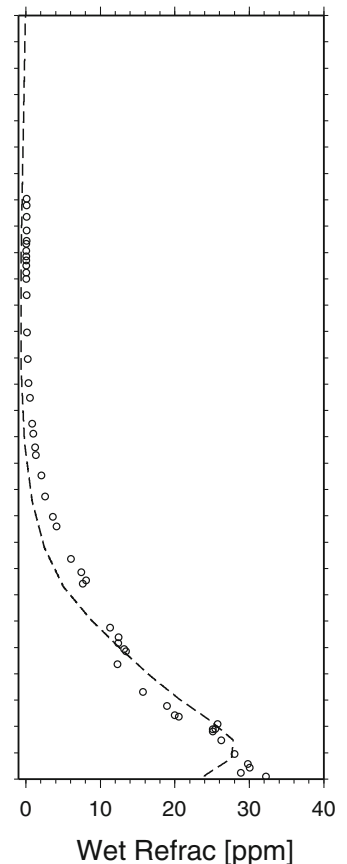

(b) trilinear, at 00h UTC

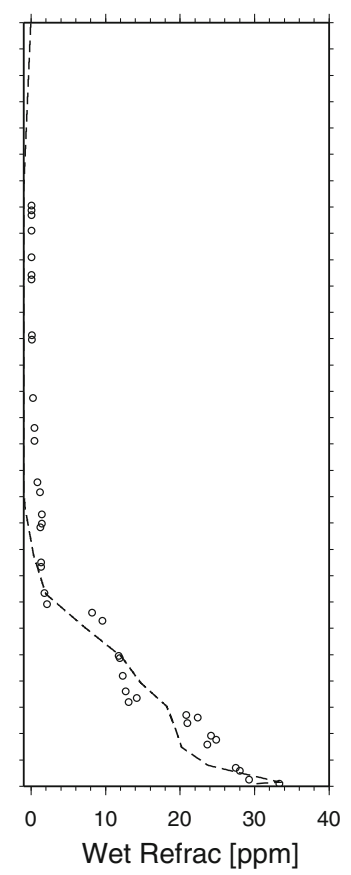

(e) trilinear, at $12 \mathrm{~h}$ UTC

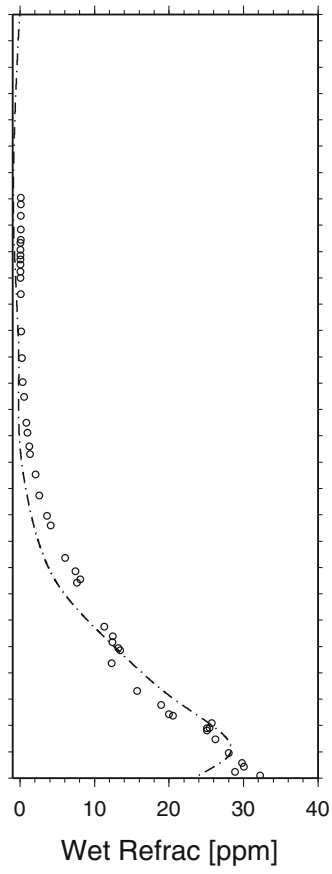

(c) bilinear/spline, at 00h UTC

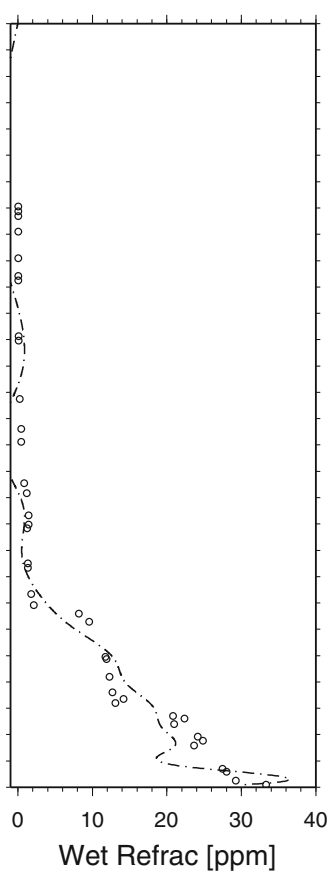

(f) bilinear/spline, at $12 \mathrm{~h} \mathrm{UTC}$ 
Table 2 Comparison from the results computed by different parameterizations to measurements of two radio sounding launches (GPS minus radio sounding)

\begin{tabular}{llll}
\hline Configuration & Bias (ppm) & Std.dev. (ppm) & IQR (ppm) \\
\hline Const 0 h UTC & -0.646 & 2.200 & 1.186 \\
Linear 0 h UTC & -0.863 & 2.137 & 1.095 \\
Spline 0 h UTC & -0.809 & 2.083 & 1.047 \\
Const 12 h UTC & -1.157 & 3.987 & 1.327 \\
Linear 12 h UTC & -1.174 & 2.069 & 1.504 \\
Spline 12 h UTC & -0.752 & 2.448 & 3.632 \\
\hline
\end{tabular}

One balloon is launched on 2006-02-26 at midnight UTC and the second $12 \mathrm{~h}$ later

low weighting factor for the pseudo-observations enables that horizontal variability can be still present in the estimated refractivity field (see e.g. Troller 2004). The regularization of the solution is the reason for the introduction of these pseudo observations.

The tomographic solutions are compared to measurements from radio soundings in Payerne (see Fig. 1). We computed tomographic results starting at 2006-02-25 $18 \mathrm{~h}$ UTC. We compared them to the balloon sondes launched at 2006-02$260 \mathrm{~h} \mathrm{UTC}$ and $12 \mathrm{~h}$, respectively. An overall good agreement between the tomographic results and the radio soundings is achieved (see Fig. 6). At midnight, all three solutions show a rather smooth decrease of refractivity with increasing height. At $750 \mathrm{~m}$ altitude, all three solutions feature an inversion which is not present in the radio sounding of 2006-02-26 0h. At noon, the solutions are more disturbed than at midnight. This is especially true for the non-parameterized and for the bilinear/spline parameterized solutions. It seems that the trilinear parameterization exerts a vertical smoothing effect. At high altitudes, regions with small negative refractivities are visible.

Ranking the different parameterizations is difficult because the statistical measures are ambivalent. The statistics are listed in Table 2. At midnight, the parameterized configuration leads to more accurate results than the nonparameterized one regarding the standard error and the IQR. At daytime, the non-parametric approach gives better results with respect to the IQR than the parameterized one. However, the non-parameterized approach contains some outliers. Therefore, the non-parametric approach has a worse accuracy than the parameterized ones regarding the standard error (see Fig. 7). For both launches, the tomographic solution has a negative bias compared to the profile above Payerne. The results show that the tomographic results reach a remarkable accuracy. For instance, the trilinear parameterized approach shows no computed value that differs more than $5 \mathrm{ppm}$ from the observations of the balloon soundings.

In comparing wet refractivity estimated from GPS phase differences with observations derived from balloon soundings, the accuracy of the balloon sounding data has

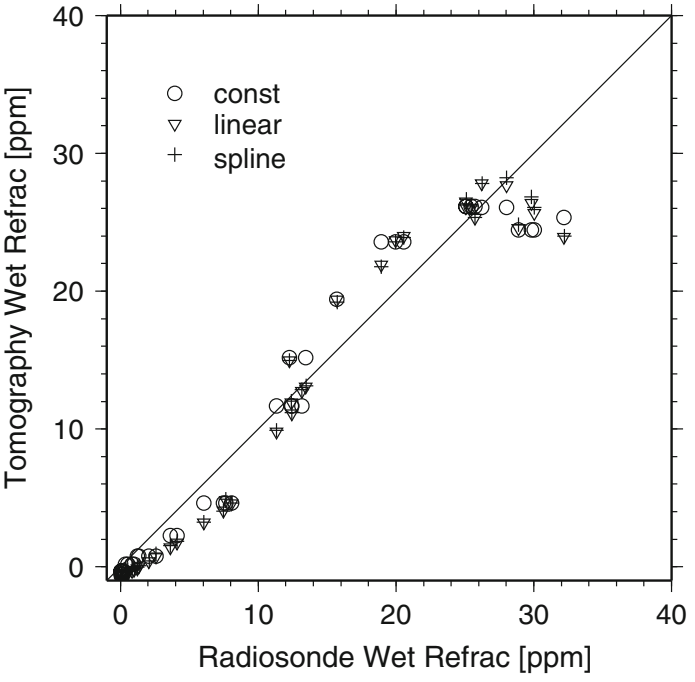

(a)

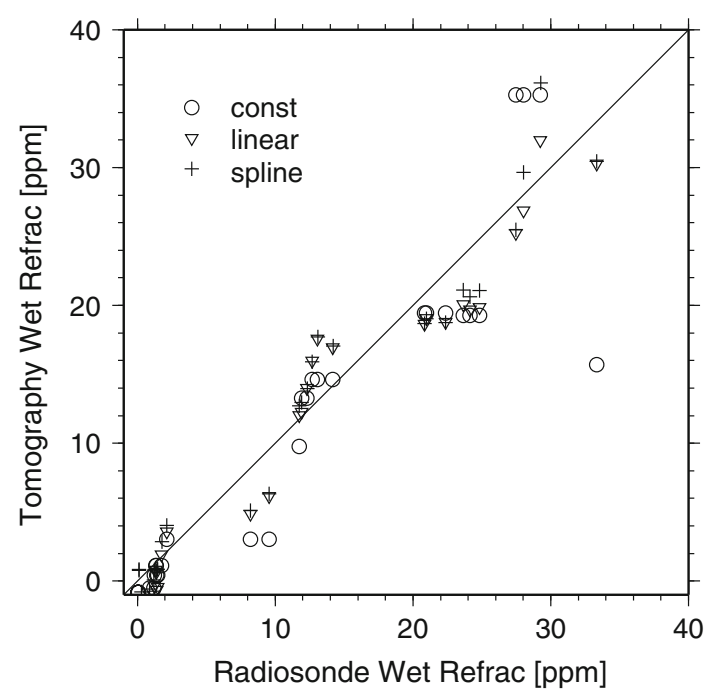

(b)

Fig. 7 Quantile-quantile-plot of tomographic solutions versus measured values retrieved from the radio soundings. a Compares data from the launch in Payerne at 2006-02-26 $00 \mathrm{~h}$ UTC and b at 2006-02-26 $12 \mathrm{~h} \mathrm{UTC.} \mathrm{a} \mathrm{2006-02-26} 00 \mathrm{~h}$, b 2006-02-26 $12 \mathrm{~h}$

to be considered, too. The accuracy of sensors mounted on balloon soundings is studied by Miloshevich et al. (2001), Wang et al. (2002), Vömel et al. (2003) and Fujiwara et al. (2003). A good overview of the accuracies is given in WMO (2008). As the wet refractivity is not directly measured by balloon soundings, it has to be computed using the wet part of Eq. 1 depending on temperature and humidity measurements. The most significant errors of temperature sensors are due to the thermal lag of the sensor, heating by solar radiation and deposition of ice or water on the sensor. The implied errors are usually below $0.5^{\circ} \mathrm{C}$ in the lower troposphere but may exceed this value when the vertical temperature gradient is large. Errors at this magnitude lead to a maximal wet 
refractivity error of about $3 \mathrm{ppm}$ assuming a temperature of $20^{\circ} \mathrm{C}$. In case of humidity sensors, wetting or icing of the sensors in clouds cause one of the most significant errors. Nash et al. (1995) state that wetting or icing leads to a relative humidity error of at least $10 \%$ on average. In wet refractivity units, this is an error up to about $10 \mathrm{ppm}$ at a temperature of $20^{\circ} \mathrm{C}$.

\section{Conclusion and outlook}

We presented new parameterized algorithms for GPS tomography in ellipsoidal coordinates and demonstrated their ability to reconstruct a four-dimensional water vapor field with high quality. These kinds of algorithms minimize discretization effects without significantly increasing the number of parameters to be estimated. Furthermore, the interpolation of in situ measurements becomes superfluous. Tests within this study indicated superior performance of the parameterized algorithms over the one without. The parameterized approaches have achieved a better accuracy of about 10-20\% in all configurations with simulated data. The bilinear/spline approach has resolved spike layers best compared to the two other approaches regarding the overall accuracy, the position of the layer, and the amplitude of the spike. Spike layer tests have indicated that vertical structures above the top station of the network can be reproduced by GPS tomography taking the curvature of the Earth into account. The vertical resolvability quickly decreases with height showing the limitations of GPS tomography in terms of vertical resolution. Layered perturbations with contributions to slant delays similar to their noise level are hardly reproduced in the experiments. This is in agreement with other studies that use real data (Troller et al. 2006; Lutz et al. 2010) where fine vertical structures are barely resolved. Besides the simulations, we also processed real data from over 40 GPS stations. Here, the parameterized approaches show superior performance compared to the non-parametric one, too. In contrast to the tests with simulated data, the most accurate solutions are obtained by the trilinear parameterization. We achieved a standard error below $2.5 \mathrm{ppm}$ in refractivity units and the maximal error was reduced from $17 \mathrm{ppm}$ with the non-parametric approach to less than $5 \mathrm{ppm}$ with the parametric approaches. All the performed validations showed the potential of the new approaches to enhance the tomographic methods. In summary, the two new approaches show similar performances. The bilinear/spline approach confirmed the superior theoretical ability to reproduce spike layers in the simulation experiments. On the other hand, the trilinear approach achieved better results with real data. This suggests that the trilinear approach is more robust than the bilinear/spline one. Further investigations are needed to decide if the simpler trilinear approach should be favored over the more complex bilinear/spline approach. Future investigations should focus on the evaluation of longer periods and on comparisons with additional test quantities like zenith wet delays. This would also allow to assess the software in terms of systematic errors. A second point to investigate is the assimilation of ground based data and other integral measurements, such as observations from radiometers, LIDARs and solar spectrometers which should increase the accuracy further.

Acknowledgments We thank D. Leuenberger and the Swiss Federal Office of Meteorology and Climatology MeteoSwiss for providing meteorological data, and E. Brockmann (Swiss Federal Office of Topography, swisstopo) for providing and processing the GPS data. We appreciate the responsible editor and the reviewer team for their valuable comments, helping us to improve the paper. This work was supported by the Swiss Federal Office of Environment (FOEN).

\section{Appendix A: Newton-Cotes quadrature}

Newton-Cotes quadrature is a numerical method to estimate a definite integral. The Newton-Cotes quadrature formulas approximate the integrand by Lagrange polynomials (Schwarz 1997; Stoer and Bulirsch 1980). In our setup, Lagrange polynomials of order 4 are used. An integral of the function $\mathrm{f}(x)$ in the interval $[a, b]$ is then approximated by

$$
\begin{aligned}
\int_{a}^{b} \mathrm{f}(x) \mathrm{d} x= & \frac{2 h}{45}\left(7(\mathrm{f}(a)+\mathrm{f}(b))+32\left(\mathrm{f}\left(x_{1}\right)+\mathrm{f}\left(x_{3}\right)\right)\right. \\
& +12 \mathrm{f}\left(x_{2}\right)+\sum_{k=1}^{N-1}\left(14 \mathrm{f}\left(x_{4 k}\right)+32\left(\mathrm{f}\left(x_{4 k+1}\right)\right.\right. \\
& \left.\left.\left.+\mathrm{f}\left(x_{4 k+3}\right)\right)+12 \mathrm{f}_{4 k+2}\right)\right)
\end{aligned}
$$

with

$h=\frac{b-a}{4 N}, \quad x_{j}=a+j h,(j=1,2, \ldots, 4 N-1)$.

\section{References}

Bastin S, Champollion C, Bock O, Drobinski P, Masson F (2007) Diurnal cycle of water vapor as documented by a dense GPS network in a coastal area during ESCOMPTE IOP2. Bull Am Meteorol Soc 46:167-182

Buzzi M (2008) Challenges in operational numerical weather prediction at high resolution in complex terrain. Ph.D. thesis, ETH Zurich

Champollion C, Masson F, Bouin M-N, Walpersdorf A, Doerflinger E, Bock O, van Baelen J (2005) GPS water vapour tomography: preliminary results from the ESCOMPTE field experiment. Atmos Res 74:253-274

Clark KM (2002) The use of computer modeling in estimating and managing future catastrophe losses. Geneva Papers Risk Insurance 27:181-195

Clark P (2009) Issues with high-resolution NWP. MOSAC-14 14.6, UK MetOffice. 12-13 November 
Dach R, Hugentobler U, Fridez P, Meindl M (eds) (2007) Bernese GPS Software Version 5.0. Astronomical Institute, University of Bern, Bern

Dankers R, Feyen L, Chrstensen OB (2009) On the benefit of highresolution climate simulations in impact studies of hydrological extremes. Hydrol Earth Syst Sci Discussions 6:2573-2597

Falconer RH, Cobby D, Smyth P, Astle G, Dent J, Golding B (2009) Pluvial flooding: new approaches in flood warning, mapping and risk management. J. Flood Risk Manage. 2:198-208

Flores A, de Arellano JV-G, Gradinarsky LP, Rius A (2001) Tomography of the lower troposphere using a small dense network of GPS receivers. IEEE Trans Geosci Remote Sensing 39(2): 439-447

Flores A, Gradinarsky LP, Elósegui P, Elgered G, Davis JL, Rius A (2000) Sensing atmospheric structure: tropospheric tomographic results of the small-scale GPS campaign at the Onsala Space Observatory. Earth Planets Space 52:941-945

Flores A, Ruffini G, Rius A (2000) 4D tropospheric tomography using GPS slant wet delays. Ann Geophys 18:223-234

Fujiwara M, Shiotani M, Hasebe F, Voemel H, Oltmans SJ, Ruppert PW, Horinouchi T, Tsuda T (2003) Performance of the meteolabor Snow White chilled-mirror hygrometer in the tropical troposphere: Comparisons with the Vaisala RS80 A/H-Humicap sensors. J Atmos Oceanic Technol 20:1534-1542

Gelb A (1974) Applied optimal estimation. MIT Press, Cambridge

Gradinarsky LP, Jarlemark P (2004) Ground-based GPS tomography of water vapor: analysis of simulated and real data. J Meteor Soc Jpn 82(1B):551-560

Hirahara K (2000) Local GPS tropospheric tomography. Earth Planets Space 52:935-939

Jacob D, Brring L, Christensen O, Christensen J, de Castro M, Dqu M, Giorgi F, Hagemann S, Hirschi M, Jones R, Kjellstrm E, Lenderink G, Rockel B, Snchez E, Schr C, Seneviratne S, Somot S, van Ulden A, van den Hurk B (2007) An inter-comparison of regional climate models for Europe: model performance in present-day climate. Clim Change 81:31-52. doi:10.1007/s10584-006-9213-4

Jehle M, Perler D, Small D, Schubert A, Meier E (2008) Estimation of atmospheric path delays in TerraSAR-X data using models vs. measurements. Sensors 8(12):8479-8491

Lutz S (2009) High-resolution GPS tomography in view of hydrological hazard assessment. Geodätisch-geophysikalische Arbeiten in der Schweiz. vol 76. Swiss Geodetic Commission

Lutz S, Troller M, Perler D, Geiger A, Kahle HG (2010) Better weather prediction using GPS. GPS World 21(7):40-47

Miloshevich LM, Voemel H, Paukkunen A, Heymsfield WJ, Oltmans SSJ (2001) Characterization and correction of relative humidity measurements from Vaisala RS80-A radiosondes at cold temperatures. J Atmos Oceanic Technol 18:135-156

Nash J, Elms JB, Oakley TJ (1995) Relative humidity sensor performance observed in recent international radiosonde comparisons. In: Ninth AMS Symposium on Meteorological Observations and Instrumentation. Charlotte, North Carolina, pp 43-48

Niell AE (1996) Global mapping functions for the atmosphere delay at radio wavelengths. J Geophys Res 101:3227-3246

Nilsson T (2005) Assessment of tomographic methods for estimation of atmospheric water vapor using ground-based GPS. Licentiate thesis, Chalmers University of Technology, Göteborg, Sweden
Nilsson T, Gradinarsky L (2006) Water vapor tomography using GPS phase observations: simulation results. IEEE Trans Geosci Remote Sensing 44(10):2927-2941

Perler D (2011) Parameterized GPS-tomography for assimilation in numerical weather prediction models. Geodätisch-geophysikalische Arbeiten in der Schweiz. Swiss Geodetic Commission (in preparation)

Pilon PJ (2005) Guidelines for reducing flood losses. United Nations

Ranzi R, Bacchi B, Ceppi A, Cislaghi M, Ehret U, Jaun S, Marx A, Hegg C, Zappa M (2009) Real-time demonstration of hydrological ensemble forecasts in Map d-Phase. La Houille Blanche, pp 95-104

Rice JA (1995) Mathematical statistics and data analysis, 2 edn. Duxbury Press, North Scituate

Rüeger JM (2002) Refractive index formulae for radio waves. In: Integration of techniques and corrections to achieve accurate engineering

Ruffini G, Flores A, Rius A (1998) GPS tomography of the ionospheric electron content with a correlation functional. IEEE Trans Geosci Remote Sensing 36(1)

Saastamoinen J (1972) Atmospheric correction for troposphere and stratosphere in radio ranging of satellites. In: Henriksen SW, Macini A, Chovitz BH (eds) The use of artificial satellites for geodesy. Geophysics monograph, vol 15. American Geophysical Union, Washington D.C. pp 247-251

Schwarz HR (1997) Numerische Mathematik, 4 edn. Teubner, Stuttgart

Stoer J, Bulirsch R (1980) Introduction to numerical analysis. Springer, New York

Troller M (2004) GPS based determination of the integrated and spatially distributed water vapor in the troposphere. Geodätisch-geophysikalische Arbeiten in der Schweiz, vol 67. Swiss Geodetic Commission

Troller M, Bürki B, Cocard M, Geiger A, Kahle H-G (2002) 3-D refractivtiy field from GPS double difference tomography. Geophys Res Lett 29(24)

Troller M, Geiger A, Brockmann E, Kahle H-G (2006) Determination of the spatial and temporal variation of tropospheric water vapour using CGPS networks. Geophys J Int 167(2):509-520

Troller M, Leuenberger D, Brockmann E, Geiger A, Kahle H-G (2007) GPS-tomography: results and analyses of the operational determination of humidity profiles over Switzerland. Geophys Res Abstr 9

Vömel H, Fujiwara M, Shiotani M, Hasebe F, Oltmans SJ, Barnes JE (2003) The behavior of the snow white chilled-mirror hygrometer in extremely dry conditions. J Atmos Oceanic Technol 20:1560-1567

Wang J, Cole HL, Carlson DJ, Miller ER, Beierle K, Laine TK (2002) Corrections of humidity measurement errors from the Vaisala RS80 radiosonde-application to TOGA COARE data. J Atmos Oceanic Technol 19:981-1002

Wanner H (1979) Zur Bildung, Verteilung und Vorhersage winterlicher Nebel im Querschnitt Jura-Alpen. Geogr. Bernensia 7, 240p G

WMO (2008) Guide to meteorological instruments and methods of observation, 7th edn. World Meteorological Organization-8, Geneva 